\title{
Knowledge, attitudes and acceptability to provider-initiated HIV testing and counseling: patients' perspectives in Moshi and Rombo Districts, Tanzania
}

RACHEL MANONGI, MICHAEL MAHANDE and BERNARD NJAU*

Kilimanjaro Christian Medical Centre, Box 3010, Moshi, Kilimanjaro, Tanzania

\begin{abstract}
Provider-initiated HIV testing and counseling (PITC) is referred to as routine testing in a clinical setting as part of a standard programme of medical services. PITC is initiated in order to avoid missed opportunities for people to get tested for HIV. While advocated as a strategy, there is dearth of information on patients' views on PITC in a number of districts in Tanzania. The objective of this study was to assess the knowledge, attitude and acceptability to PITC services among patients attending health care facilities in rural and urban settings in Kilimanjaro region A total of 12 focus group discussions (FGDs) were conducted with 99 (73 female and 26 male) patients enrolled into outpatient clinics in 8 ( 2 hospitals and 6 primary care centers) health facilities in Moshi Urban and Rombo districts in northern Tanzania. The study explored on knowledge, attitudes and acceptability of PITC, perceived benefits and barriers of PITC, and ethical issues related to PITC. Interviews were audio taped, transcribed, translated, and analyzed using Non-numerical Unstructured Data Indexing and Theorizing (NUDIST) software. Knowledge about PITC services was generally low. Compared to men, women had a more positive attitude towards PITC services, because of its ability to identify and treat undiagnosed HIV cases. HIV stigma was regarded as a major barrier to patients' uptake of PITC. Institutional factors such as lack of supplies and human resources were identified as barriers to successful provision of PITC. In conclusion, the findings highlight both opportunities and potential barriers in the successful uptake of PITC, and underscore the importance of informed consent, counseling and confidentiality and the need for specific strategies on advocacy for the service.
\end{abstract}

Keywords: HIV, knowledge, attitude, acceptability, counseling, testing, provider-initiated, Tanzania

\section{Introduction}

HIV voluntary counseling and testing (VCT) services have become well established as an essential component of many AIDS control programmes and a cost-effective means of HIV prevention globally (UNAIDS, 2008; Wringe et al., 2008). There have been major advances in the provision of health care services to HIV-infected people in Africa (UNAIDS, 2008,2012,2013), including Tanzania (MoHSW, 2007; Roura et al., 2008; THMIS, 2013), and most importantly, the increasing accessibility of antiretroviral therapy (ART). Despite the increased emphasis on VCT, and availability of ART and other health care services for HIV-infected patients in sub-Saharan Africa, many cases of HIV go undiagnosed (UNAIDS, 2014). These "missed opportunities" for diagnosis may be best filled by offering routine HIV testing to all patients in the health care settings. However, these opportunities among patients who attend health care services have received relatively little attention (Turan et al., 2008; UNAIDS, 2008).

To address the 'missed opportunity' the World Health Organization (WHO) initiated Provider Initiated Testing and Counseling (PITC) programme. PITC is referred to as a routine testing in a clinical setting as part of a standard programme of medical services (UNAIDS, 2008). In PITC, health care providers offer testing during the patient evaluation with full adherence to informed consent, confidentiality and counseling whereby patients have the right to accept or refuse to be tested. The aim of this initiative was to scale up HIV counseling and testing (HCT) services at clinical settings, fight stigma and discrimination and thus reduce the fearful nature of the HIV epidemic (UNAIDS, 2008;Meiberg et al.,2008).

\footnotetext{
Correspondence: Bernard Njau; E-mail: biesein2007@gmail.com
} 
While the Tanzania Government through the Ministry of Health and Social Welfare has adopted the programme, there are few insights into patient's perspectives on PITC services among patients attending health care facilities in Tanzania (MoHSW, 2007). The objective of this study was therefore, to assess the knowledge, attitude and acceptability to PITC services among patients attending health care facilities in Moshi Urban and Rombo districts of northern Tanzania. Understanding the perspectives of patients on PITC services can provide insight into how best to implement effective, efficient and user friendly PITC services in these settings.

\section{Materials and Methods}

\section{Study area and data collection}

This study was carried out in Moshi Urban and Rombo Districts of northern Tanzania. Data was collected using 12 focus group discussions (FGDs) conducted in February 2008. Two hospitals (one public and one private) and six dispensaries that serve approximately 445,255 people were involved in the study. A purposeful sample was used in order to get representation by age and sex. All participants were at least 18 years old and able to provide informed consent.

Trained researchers conducted the FGDs with eligible patients in Kiswahili language. The interview covered knowledge, attitudes and acceptability of PITC. Other information explored were ethical issues related to PITC, benefits and barriers of PITC, and recommendations for increasing uptake of the services. The FGDs were conducted at the end of patients' consultations with their primary care providers. FGDs were tape-recorded, lasted between 60 to 90 minutes and were later transcribed by the researchers with full adherence to patient confidentiality.

\section{Data analysis}

An independent translator translated each Kiswahili transcript into a hand-written English transcript. A framework approach (Spencer et al., 2003; Singleton \& Straits, 2005) combining the use of Non-numerical Unstructured Data Indexing and Theorizing (NUDIST) software and manual techniques guided the identification of key themes used to develop the thematic framework, which was systematically applied to sort data. Translated transcripts were coded by all the investigators and an independent qualitative analyst verified the coded data. Discrepancies in coding were discussed among investigators and resolved. The texts were read repeatedly to identify major themes and each theme was broken down into concepts. Representative, verbatim quotes were selected to illustrate key findings.

\section{Ethical considerations}

The study received ethical approval from Kilimanjaro Christian Medical College Research Ethics Committee. All respondents were informed of the study aims, confidentiality and procedures for verbal consent.

\section{Results}

\section{Knowledge and attitudes to PITC}

A total of 99 respondents were involved in the study (46 in rural; 53 in urban settings). Most respondents lacked knowledge of PITC services. It was very common for respondents to use the words "hatujui inamaanisha nini" meaning, "we don't know the meaning" of PITC. However, in an urban health facility, some informants were able to describe the main components of PITC services. One of the discussant attending an out-patient clinic had this to say: "It is the health care provider, who initiates counseling and testing and later gives advice to those who are found (HIV) positive on how to live positively" (Male patient, urban health facility).

Respondent's attitude towards PITC seemed to be influenced by better treatment options and care from health care providers after testing for HIV. In most discussions, informants 
spoke of the right of a sick individual to ask for voluntary counseling and testing. One female discussant in rural health facility observed: "If a health care provider initiates counseling and testing it is not bad, it is because s/he wants to know how best to treat you whether it is malaria or HIV" (Female patient, rural health facility). On the other hand, a male patient at an urban health facility underscored the right of patients to request for voluntary counseling and testing, "Even an individual who is sick can ask for counseling and testing to know his/her health status".

\section{Acceptability of PITC}

Acceptability of PITC was dominated by fear and stress involved with HIV testing, particularly among male respondents. Most men expressed that health care providers should only initiate PITC when a person is sick. Some men had the following opinion on acceptability to PITC as narrated: ".... my comment is that it will be very bitter for me when I come to see a doctor for a certain problem and she or he starts telling me about checking for HIV the virus" (Male patient, urban health facility). Fear accompanied with stress was mentioned by most male respondents. The stressful experiences accompanying HIV testing is well described by the following narrative: “... when the health care provider tells you to check your health status (HIV testing) you become desperate with no peace of mind. You will think that she or he has doubts about your health. A patient will think that she is already infected that is why is being asked to check for HIV. The person will start getting worries as to why she or he is being asked to check for HIV status" (Male patient, urban health facility). Further, another male discussant perceived that PITC may discourage people from seeking health care services: "I think that if the doctor decides to ask everybody to test then she/he will discourage people from coming to hospital and people will die at home" (Male patient, urban health facility).

Contrary to male respondents, women had more positive views regarding acceptability to PITC. Most female respondents observed that the act of health care providers initiating HIV testing services was seen as a positive idea: "It is true because last year I attended a hospital for treatment of malaria but the nurse advised me to also check for HIV. Initially I was shocked but after being counseled, I accepted to test and received my results. I was really happy after being found negative and until today I am living with confidence than I did before testing" (Female patient, rural health facility). Female respondents expressed that PITC will assist in early initiation of treatment and care and save patients' lives, "I think it is better if doctors initiate testing because you will know your status and if positive you will get services earlier and hence live a long life" (Female patient, urban health facility). Another discussant wondered, "How will a doctor save a patient if she does not take all tests?" (Female patient, urban health facility).

\section{Ethical issues}

Patients had strong opinions on ethical issues related to HIV testing and counseling. Most informants underscored the importance of counseling, consent and confidentiality and that PITC should be done on voluntarily basis: "I advise that the doctor should tell you straight away that you are going to check for HIV; and get prepared if you are going for HIV test" (Female patient, urban health facility). A male discussant added that: "When you go to hospital it is better that a person makes her/his own decision. A doctor may only initiate testing and counseling of the client" (Male patient, urban health facility).

In most discussions, respondents had a high opinion on health care providers' level of confidentiality. Both male and female discussants praised health care providers' for their high standards of confidentiality. However, in urban settings some respondents mentioned breaches of confidentiality among health care providers. A female discussant had this to say: "There is high confidentiality because we [patients] have not heard any patient's result being disclosed. Health care providers always assure us about confidentiality during counseling" (Female patient, rural health facility; Male patient, urban health facility). 
In most discussions in rural settings, respondents agreed that health care providers attend their patients well. A female discussant had these to say on her perception towards health care providers: "Health care providers receive us nicely and use polite language; most of them are very sympathetic to their patients" (Female patient, rural health facility).

Some patients attending urban health facilities observed that HIV testing was not always practiced as a voluntary decision. Patients blamed some health care providers for not adhering to voluntary HIV testing. A female patient gave a following narrative: "It is practiced in this hospital, one day I had stomachache and was told to draw blood for testing (HIV test) without being counseled" (Female patient, urban health facility). A male discussant said the following: "Lack of confidentiality among some health care providers is a barrier for HIV testing. Some clients are worried to go for HIV testing because they are well known to some health workers" (Male patient, urban health facility).

\section{Benefit of PITC}

Patients' expectations about PITC were influenced by the opportunity for earlier detection, early initiation of treatment and care, and introduction of risk-reduction strategies. In most FGDs, most discussants had opinions that PITC will assist in stigma reduction associated with HIV testing. The advantage of early detection of HIV was well illustrated by a female patient attending out-patient clinic in urban setting: "It is not good to live with worries, just test and if you are HIV-positive they will tell you what to do". Another narrative on the same issue was provided by a female discussant in rural settings: "It has more advantages, for example, if a person has been sick for a long time and does not get better in spite of treatment, then it is appropriate to initiate such a service (PITC).

Risk-reduction was also highlighted by most informants as one of the benefits of PITC services. This observation was more common among female respondents as illustrated in the following narrative: "The counseling that a man gets will be useful. If he [client] is a promiscuous it will help him to correct his behaviour and if he is HIV infected, he will be counseled" (Female patient, Moshi urban).

Female respondents also showed that PITC is an entry point in knowing the HIV status among couples: "Personally, I think a person [wife] lives with worries without knowing what goes on with her husband when he is away [....]. If you check [HIV testing] as a couple, you will know your health status. ... .if you are positive then you become aware of yourself. It is easy if health workers act as catalyst in counseling client to test for their HIV status (Female patient, urban health facility).

Most respondents agreed that PITC will reduce the time spent visiting a specialized VCT clinic, as HIV testing and counseling will be provided during a routine clinic visit. This benefit was well documented by a female patient at a rural health facility, who said: "It is possible that a person accompanying a patient to the hospital has limited time. Therefore it is good if he/she tests because he/she may not get another chance. If he is informed about counseling and testing to check his health status it will be an advantage to him" (Female patient, rural health facility).

\section{Barriers to PITC}

Most respondents mentioned barriers, which may hinder a successful uptake of PITC services. Individual barriers related to patients' uptake of PITC included stigma and discrimination, lack of HIV sero-status disclosure among couples, and lack of knowledge on HIV transmission. Health systems-related barriers included absence of health care providers, lack of HIV testing facilities, lack of drugs and longer distances to health facilities. A female discussant observed: "Unavailability of HIV testing equipment or supplies or ARV or drugs for treating opportunistic diseases at peripheral health facilities can reduce uptake of HIV testing. "Sometimes you may go to a health facility and find that the clinician is not present and you have to wait for her/him" (Female patient, rural health facility). 
Fear of death after testing positive for HIV, outcomes related to HIV-positive disclosure and lack of knowledge on HIV transmission were mentioned by most respondents as barriers related to stigma and discrimination associated with HIV/AIDS. A male discussant attending a rural health facility observed, "Most people are afraid to test for HIV and once they know they think that they are going to die" (Male patient, rural health facility).

Acceptance of a sero-positive partner was also mentioned as a significant factor fuelling stigma and discrimination associated with HIV testing. One discussant attending an out-patient clinic had these to say: "Stigma is a major problem in the community, especially among couples; it is very difficult to accept a sero-positive partner, as this is associated with being unfaithful in marriage" (Female patient, rural health facility).

HIV positive people are still marginalized in their communities. The most common explanation for the marginalization was mentioned as lack of knowledge about HIV transmission. A female discussant attending a rural health facility said: "Some people in the community still marginalize HIV-positive individuals; this is a result of ignorance about HIV transmission. For an example, there are people who believe that they can be infected if they eat together with an infected person".

\section{Lessons for implementation of PITC uptake}

Advocacy for PITC services, as well as couple counseling services, were viewed as essential components for a successful uptake of PITC services. Advocacy for PITC may be achieved through health education campaigns in both clinics and community settings. Most respondents had an opinion that patients may be educated through seminars or drama at the clinic and the community levels. This was clearly captured in the following narrative from a male discussant attending out-patient clinic at a hospital in urban setting: "I plead that health care providers educate people before testing them for HIV infection. A female patient attending an out-patient clinic at an urban health facility suggested that seminars and drama at the community level can assist in advocacy for PITC services: "Seminars (on PITC) should be conducted; drama in which people act the role of those men who don't want to be tested drama will be useful".

The majority of female discussants in both rural and urban settings had opinions that males have a major role to play in the advocacy for PITC services. Most female patients blamed their male partners/husbands for not using VCT services. A female discussant at an out-patient clinic at urban health facility said: "They (men) persuade women to come for testing and some women do not come until when they are pregnant. Since all pregnant women are required to test health care providers should take the opportunity and ask pregnant mothers to come with their husbands. Accompanying their wives could help solve the problem of low HIV testing rate among men". Another female informant asked for the need to have a law or regulation that oversees couple counseling: "There should be law that all pregnant mothers attending the PMTC clinic should be accompanied by their partners/or husbands so that they can test together".

Women acknowledged their role in influencing their male partners in PITC uptake. Most female patients in urban health facilities had an opinion that women are more likely to meet in groups or attend meetings and later on share their experiences with their partners. This scenario was well described by a female discussant attending an out-patient clinic in an urban health facility as follows: "It is not possible to meet men in groups, men are difficult. But women like group meetings/or gatherings and can be sensitized and then educate their husbands when get back home Men are difficult but if you remind them regularly, they may soften".

Couple counseling was viewed by most respondents, particularly females, as an essential component of PITC and a way to possibly increase uptake of the service. Some female discussants wondered how female patients can test for HIV without informing their husbands. The importance of couple counseling among sick patients who attend out-patient clinics was highlighted by a female discussant attending a hospital in urban health facility. She gave her views as follows: "In my views I would ask that when the woman is sick she should go to the 
doctor with her husband; this would make easier for the doctor to advise both of them to check for their health status"

\section{Discussion}

The findings presented here demonstrate that patients enrolled in public sector out-patient health services in Moshi and Rombo districts have inadequate knowledge about PITC services. This apparent low knowledge may be attributed to the fact that PITC service is a new concept in the country (MoHSW, 2007). The observation of low knowledge underscores the importance of advocacy on PITC at all levels of health care delivery system in the two districts, as well as the community at large, as suggested by respondents in this study. Despite respondents' initial low knowledge about PITC services; they expressed strong opinions about the perceived acceptability, benefits and barriers of the services, informed in part by exposure to traditional VCT and PMTCT services.

In general, respondents had a positive attitude towards PITC services in both urban and rural settings, with female respondents expressing most favorable opinions. Most female respondents said that the act of health care providers initiating HIV-counseling and testing was beneficial, especially for early initiation of treatment and care. Also, patients' right to request for voluntary counseling and testing was influential for the observed positive attitude towards PITC services. On the other hand, although PITC services were considered acceptable by patients, male respondents expressed fear and stress involved with HIV testing, in particular when the testing is not planned or anticipated. Several studies on counseling and testing have observed similar findings of stressful experiences accompanying HIV- testing (Osterman et al., 2011; Mukolo et al., 2013; Musheke et al., 2013; UNAIDS, 2012,2013).

One respondent suggested that initiation of PITC can reduce health care seeking behaviours of patients, because of fear of being identified as having HIV or AIDS. Although this did not come up repeatedly, the observation concurs with findings in studies by others elsewhere (Matovu \& Makumbi, 2007; Batengaya et al., 2010; Njau et al., 2012; Suthar et al., 2013). Respondents' hesitation about routine HIV testing in the clinic setting suggests a need for addressing fear and stressful experiences during HIV-testing within the health care system, especially among men. It is important for all providers to create a 'safe' and supportive environment and become familiar with the concerns of HIV-positive persons within the health care and the social environment (Shorter et al., 2009; Bategaya et al., 2010; Osterman et al., 2011; Suthar et al.,2013).

Overall the introduction of PITC services initiated by health care providers is very promising, particularly among female respondents. This observation can be associated with the PMTCT services, which provides opportunity for pregnant women to have access to HIV-related information and HCT services (Becker et al., 2010; Hood et al., 2012; Kennedy et al., 2013), and has also been associated with higher uptake of HIV-counseling and testing among women (Turan et al., 2008; Becker et al., 2010). In addition, women are the primary care-taker of sick children in the community, so much so that the health care centre literally becomes a second home. In this way, the woman may build confidence and trust towards health providers, to the extent that she will have full trust on any advice given by health providers ( UNAIDS 2012, 2013).

Patients had strong opinions about ethical issues related to voluntary HIV-testing and counseling. Issues regarding the importance of informed consent, counseling and confidentiality emerged in most discussions in both rural and urban settings. Ethical issues related to HIV counseling and testing are very sensitive and providers are expected to be aware of ethical and legal implications while discharging PITC services (Angotti et al., 2009; Ostermann et al., 2011). This is necessary because of the sensitive nature of HIV infection, associated stigma and vulnerability of clients. PITC services demand a high degree of confidentiality and trust in order to achieve good cooperation from the public (UNAIDS, 2013). Further, PITC services must adhere to 
the principles of consent and confidentiality, and the opt-out alternative in order to safeguard privacy, integrity and right to self determination of individuals. For most patients, health care providers' attitude towards their patients and use of friendly language was perceived to play a complimentary role with clinical care in respondents' lives. Opportunity for earlier detection, early initiation of treatment and care and risk reduction strategies provided by PITC services were perceived highly by most respondents. The benefits of VCT services have been documented elsewhere by Anderson et al. (2008), Shorter et al. (2009), Mukolo et al. (2013) and Musheke et al. (2013. They have also been observed as entry points into couple counseling and increased HIV sero-status disclosure among couples (Becker et al., 2010; Kelley et al., 2011; Njau et al., 2012). This observation therefore calls for health care providers to maintain high level of confidentiality and patient care and explore the opportunity for couple counseling and promote disclosure among couples.

Integrating HIV- counseling and testing into the routine health care services was regarded as an efficient approach to care. In fact most respondents agreed that PITC services will reduce stigma and the time spent visiting a specialized VCT clinic, as HIV testing and counseling will be provided during a routine clinic visit. A visit to a specialized facility just for an HIV test can be time consuming, inconvenient and stigmatizing. PITC takes less time, since the focus is more on post test counseling and referral to appropriate services (Batengaya et al., 2010; Musheke et al.,2013; Mukolo et al., 2013; Kennedy et al., 2013). This observation underscore the importance of integrating PITC as one of the components of a comprehensive strategy to make HIV testing widely available within the health system. However, these efforts should not jeopardize the continued scaling-up of VCT, beyond the health services (Suthar et al., 2013; UNAIDS, 2013).

Barriers to PITC services were categorized into individual and health care services related. Respondents revealed that stigma and discrimination exerted negative influences by expressing judgmental attitudes about HIV-positive people. Stigma and discrimination associated with HIV/AIDS have been documented in many settings, in large part because of the perceived relation between HIV and "immoral" sexual behaviour (Wringe et al., 2008; Ulasi et al., 2009; Musheke et al., 2013). The stigma associated with the testing hinders many to come forward for HCT. PITC services are thought to fill the 'missed opportunity' gap in clinical settings, as health care provider initiate routine HIV testing as part of a standard programme of medical services. By initiating counseling and testing during a routine health service visit, all patients may reduce stigma associated with a HIV diagnosis.

While health care facilities play an important role in providing the clinical needs related to HIV epidemic in Tanzania (MoHSW, 2008; TACAIDS, 2012; THMIS, 2013), absenteeism of health care providers and lack of HIV testing equipment or supplies or drugs for opportunistic diseases, particularly in primary care centers, are recognized as major barriers to an efficient PITC programme (Ntuli et al., 2011; Kennedy et al., 2013). This echoes findings in other parts of Africa whereby the existing health care systems are failing to cope with the increased burden of HIVrelated health care needs (Batengaya et al., 2010; Ntuli et al., 2011; Kennedy et al., 2013; Musheke et al., 2013). In addition, long distance to health facilities was mentioned as a barrier to PITC services. This observation therefore underscores the importance of scaling-up PITC to primary care centers, such as dispensaries.

The study's findings highlight opportunities for advocacy for PITC services in both urban and rural settings. Most respondents viewed advocacy for PITC services as an essential component for a successful PITC uptake. Health education campaigns through seminars and drama were regarded as the most effective approach in sensitization of PITC services. However, caution must be taken to avoid prevention messages that equate immoral behavior with HIV, which may inadvertently enforce discrimination against HIV-positive persons (Meiberg et al., 2008; Roura et al., 2009; Ntuli et al., 2011; Musheke et al., 2013). Instead, an environment should be created in which people living with HIV/AIDS (PLWHA) will feel comfortable to disclose their sero-status without fear. Further, the environment can be strengthened by inviting PLWHA to 
speak at clinical and community settings, in order to introduce role models for positive living and disclosure (Turan et al., 2008; Wringe et al., 2008).

Finally, men have an important role to play in advocacy for HIV counseling and testing, including PITC. Males should be encouraged to seek out counseling and testing, together with their spouses/or partners through couple counseling strategy (Msuya et al., 2008; Wringe et al., 2008; Bwambale et al., 2008; van Rooyen et al., 2013). Most female respondents in this study blamed their male partners/or husbands for low uptake of HIV testing. The observation concurs with findings of a similar study by Msuya et al. (2008). In 2004, the UNAIDS World AIDS campaign, highlighted the need to target men in the effort to contain the spread of HIV epidemic (UNAIDS, 2004). This observation calls for efforts to involve men in advocacy for PITC, in order to reduce the low HIV-testing uptake among them and increase HIV-sero-status disclosure rates among sexual partners (Bwambale et al., 2008; van Rooyen et al., 2013).

The results presented in this study must be viewed in light of the study's limitations. Despite our efforts to recruit a wide range of participants, the men and women who agreed to participate may differ in significant ways from those who chose not to participate. In addition, the themes reported in this study are limited to opinions that individual respondents chose to share during the discussions. The nature of qualitative study may have limited respondents from discussing more sensitive issues with candor and honesty. Finally, the convenience sampling approach, the fact that all participants were recruited from a health care setting, and exclude the community setting made it difficult to generalize the study findings to other settings. Despite the limitations of the study, the results provide very important insight into the perspectives of patients enrolled in public health care sector on PITC services in urban and rural settings in northern Tanzania. The findings highlight both opportunities and potential barriers in the successful uptake of PITC, and underscore the importance of informed consent, counseling and confidentiality and the need for specific strategies on advocacy for PITC services.

\section{References}

Anderson, M., Elam, G., Gerver, S., Solarin, I., Fenton, K. \& Easterbrook, P. (2008) HIV/AIDSrelated stigma and discrimination: accounts of HIV-positive Caribbean people in the United Kingdom. Social Science \& Medicine 67, 790-798.

Angotti ,N., Bula ,A., Gaydosh, L., Kimchi, E. Z., Thornton, R.L. \& Yeatman, S.E. (2009) Increasing the Acceptability of HIV counseling and testing with three C's: convenience, confidentiality and credibility. Social Science \& Medicine 68, 2263-70.

Becker, S., Mlay, R., Schwandt, H.M. \& Lyamuya, E. (2010) Comparing couples' and individual voluntary counseling and testing for HIV at antenatal clinics in Tanzania: a randomized trial. AIDS and Behavior 14, 558-566.

Bwambale, F.M., Ssali, S.N., Byaruhanga, S., Kalyango, J.N. \& Karamagi, C.A. (2008) Voluntary HIV counselling and testing among men in rural western Uganda: implications for HIV prevention. BMC Public Health 8:263.

Hood, J.E., Mackellar, D., Spaulding, A., Nelson, R., Mosiakgabo, B., Sikwa, B,Puso,I., Raats, J., Alwano, G.M. \& Monyatsi, B. (2012) Client characteristics and gender-specific correlates of testing HIV positive: a comparison of stand alone center versus mobile outreach HIV testing and counseling in Botswana. AIDS and Behavior 16, 1902-1916.

Kelley, A.L., Karita, E., Sullivan, P.S., Katangulia, F., Chomba, E., Carael, M., Telfair, J., Dunham, S. M., Vwalika, C.M., Kautzman, M.G., Wall mail, K.M. \& Allen, S.A. (2011) Knowledge and perceptions of couples' voluntary counseling and testing in urban Rwanda and Zambia: a cross-sectional household survey. PLoS One 6(5):e19573.

Kennedy, C.E., Fonner, V.A., Sweat, M.D., Okero, F.A., Baggaley, R. \& O'Reilly, K.R. (2013) Provider initiated HIV testing and counseling in low- and middle-income countries: a systematic review. AIDS and Behavior 17, 1571-1590. 
Matovu, J.K. \& Makumbi, F.E. (2007) Expanding access to voluntary HIV counseling and testing in sub-Saharan Africa: alternative approaches for improving uptake, 2001-2007. Tropical Medicine \& International Health 12, 1315-1322.

Meiberg, A.E., Boss, A.E., Onya, H.E., \& Schaalma, H.P. (2008) Fear of stigmatization as barrier to voluntary HIV counselling and testing in South Africa. East African Journal of Public Health 5, 49-54.

MoHSW (2007) Guidelines for HIV Testing and Counseling in Clinical settings. United Republic of Tanzania, Ministry of Health and Social Welfare. Dar es Salaam (Available at: http//:www.tanzania.go.tz/).

MoHSW (2008) Health Sector Performance Profile Report, Mainland Tanzania July 2006 - June 2007. United Republic of Tanzania, Ministry of Health and Social Welfare, Dar es Salaam.

Mukolo, A., Villegas, R., Aliyu, M. \& Wallston, K.A. (2013) Predictors of late presentation for HIV diagnosis: a literature review and suggested way forward. AIDS and Behavior 17, 5-30.

Musheke, M., Ntalasha, H., Gari, S., McKenzie ,O., Bond, V., Martin-Hilber, A. \& Merten, S. (2013) A systematic review of qualitative findings on factors enabling and deterring uptake of HIV testing in Sub-Saharan Africa. BMC Public Health 13:220.

Msuya, S.E., Mbizvo, E.M., Hussain, A., Uriyo, J., Sam, N.E., \& Stray-Pedersen, B. (2008) Low male partner participation in antenatal HIV counseling and testing in northern Tanzania: implications for preventive programs. AIDS Care 20, 700-709.

Njau, B., Watt, M.H., Ostermann, J., Manongi, R., Sikkema, K.J. (2012) Perceived acceptability of home-based couples voluntary HIV counseling and testing in northern Tanzania. AIDS Care 24, 413-419.

Ntuli, A.K., Kabengula, J.S. \& Msuya, S.E. (2011) Perceived barriers and attitudes of health care providers towards Provider-Initiated HIV Testing and Counseling in Mbeya region, southern highland zone of Tanzania. The Pan African Medical Journal 8:17.

Ostermann, J., Reddy, E.A., Shorter, M.M., Muiruri, C., Mtalo, A., Itemba, D.K, Njau, B., Bartlet, J.A., Crump, J.A. \& Thielman, N.M. (2011) Who Tests, Who Doesn't, and Why? Uptake of Mobile HIV Counseling and Testing in the Kilimanjaro Region of Tanzania. PLoS One. 6(1):e16488.

Roura, M., Urassa, M., Busza, J., Mbata, D., Wringe, A. \& Zaba, B . (2009) Scaling up stigma? The effects of antiretroviral roll-out on stigma and HIV testing. Early evidence from rural Tanzania. Sexually Transmitted Infections 85, 308-312.

Singleton, R.A. \& Starits, B.C. (2005) Approaches to social research ( $4^{\text {th }}$ edn). Oxford University Press, new York, USA.

Suthar, A.B., Ford, N., Bachanas, P.J., Wong, V.J., Rajan, J.S., Saltzman, A.K., Ajose, O., Fakoya, A. O., Granich, R.M., Negussie, E.K. \& Baggaley, R.C. (2013) Towards universal voluntary HIV testing and counseling: a systematic review and meta-analysis of community-based approaches. PLoS Medicine 10(8):e1001496.

Spencer, L., Richie, J. \& O'Connor, W. (2003) Carrying out qualitative analysis. In J. Ritchie \& J. Lewis (Eds.), Qualitative Research Practice: A guide for Social Science Students and Researchers (pp.219-262). London : Sage.

Shorter, M.M., Ostermann, J., Crump, J.A., Tribble, A.C., Itemba, D.K., Mgonja, A., Mtalo, A., Bartlett, J.A., Schimana, W. \& Thielman, N.M. (2009) Characteristics of HIV voluntary counseling and testing clients before and during care and treatment scale-up in Moshi,Tanzania. Journal of Acquired Immune Deficiency Syndrome 52, 648-654.

TACAIDS (2012) UNGASS Country Progress Reporting - Tanzania Mainland 2012 [30-Mar-2012]. Available at: http://www.unaids.org/en/dataanalysis/

THMIS (2013) Tanzania HIV/AIDS and Malaria Indicator Survey 2011-12 Dar es Salaam, Tanzania. Tanzania Commission for AIDS, Zanzibar AIDS Commission, National Bureau of Statistics, Office of the Chief Government Statistician and Macro International Inc. 
Turan, J., Miller, S., Bukusi, E. A., Sande, J. \& Cohen, C. R. (2008) HIV/AIDS and maternity care in Kenya: how fears of stigma and discrimination affects uptake and provision of labor and delivery services. AIDS Care 20, 938-45.

Ulasi, C.I., Perko, P.O., Baidoo, J.A., Buyard, B., Ehiri, J.E., Jolly, C.M. \& Jolly, P.E. (2009) HIV/AIDSrelated stigma in Kumasi, Ghana. Health and Place 1, 255-262.

UNAIDS (2004) Global Report: UNAIDS Report on the Global AIDS Epidemic 2004. Geneva, Switzerland. www.unaids.org/.../unaids/.../unaidspublication/2004/GAR2004_en.pdf.

UNAIDS (2008) Global Report: UNAIDS Report on the Global AIDS Epidemic 2008. Geneva, Switzerland

UNAIDS (2012) Global Report: UNAIDS Report on the Global AIDS Epidemic 2012. Geneva, Switzerland

UNAIDS (2013) Global Report: UNAIDS Report on the Global AIDS Epidemic 2013. Geneva, Switzerland.

UNAIDS (2014) UNAIDS Gap Report 2014, Geneva, Switzerland. 2014. www.unaids.org/en/.../unaids/.../unaidspublication/2014/GAR2014_en.pdf.

van Rooyen, H., McGrath, N., Chirowodza, A., Joseph, P., Fiamma, A., Gray, G., Richter, L. \& Coates, T. (2013) Mobile VCT: reaching men and young people in urban and rural South African pilot studies (NIMH Project Accept, HPTN 043). AIDS and Behavior 17, 2946-2953.

Wringe, A., Isingo, R., Urassa, M., Maiseli, G., Manyalla, R., Changalucha, J., Mngara, J., Kalluvya, S., Zaba, B. (2008) Uptake of HIV voluntary counselling and testing services in rural Tanzania: implications for effective HIV prevention and equitable acces to treatment. Tropical Medicine \& International Health 13, 319-327. 